\title{
Termorregulação de frangos de corte alimentados com dietas contendo níveis de grãos secos de destilaria
}

\author{
Thermoregulation of broilers fed with diets containing dried distillers \\ grain levels
}

\begin{abstract}
Jean Kaique Valentim (1) ${ }^{1 *}$, Tatiana Marques Bittencourt (10 2), Heder José D'Ávilla Lima (1) 2, Debora Duarte

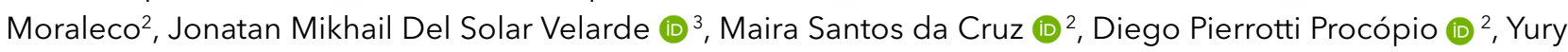
Gonçalves Lopes², Rodrigo Garófallo Garcia (10 ${ }^{1}$
\end{abstract}

${ }^{1}$ Universidade Federal da Grande Dourados (UFGD), Dourados, MS, Brasil

2 Universidade Federal de Mato Grosso (UFMT), Cuiabá, MT, Brasil

${ }^{3}$ Universidade Federal do Sergipe (UFS), São Cristovão, SE, Brasil

\section{Resumo}

O objetivo deste estudo foi determinar os efeitos da inclusão de grãos secos de destilaria (DDG) de milho sobre os parâmetros de termorregulação de frangos de corte. Utilizaram-se 700 frangos de corte da linhagem Coob 500, machos, com 1 dia de idade, distribuídos em um delineamento inteiramente casualizado com cinco tratamentos, sete repetições e dez aves por unidade experimental. Os níveis de DDG estudados foram 4, 8, 12 e 16\% de inclusão nas dietas, além do tratamento controle sem inclusão. No final de cada fase de produção (7, 28 e 42 dias de idade) foram coletados os dados relacionados às variáveis termorregulatórias das aves. As médias obtidas a partir dos parâmetros avaliados foram submetidas à análise de variância a
$5 \%$ de probabilidade, utilizando-se o programa SAS. O índice de temperatura e umidade (ITU) se apresentou na faixa de 68,49 a 71,65 durante o tempo de produção. Não houve efeito $(p>0,05)$ para as variáveis, temperatura cloacal, temperatura média corporal, temperatura média da pele e temperatura média da cama de frangos de corte aos 7, 35 e 42 dias de idade, alimentados com dietas com níveis de DDG de milho na dieta. $O$ uso de DDG em dietas de frangos de corte não influenciou as variáveis de termorregulação das aves.

Palavras-chave: Avicultura de corte. Coprodutos. Temperaturas corporais. 


\section{Abstract}

The objective of this study was to determine the effects of the inclusion of dry beans to a distillery (DDG) of corn on thermoregulation of broilers. Seven hundred Coob 500 male chicks with 1 day of age were used and distributed into a completely randomized design with five treatments, seven repetitions, and ten broilers per experimental unit. The designed treatments consisted of the inclusion of $4,8,12$ and $16 \%$ corn DDG, in addition to the control treatment without inclusion. At the end of each production stage (7,28 and 42 days old), data related to thermoregulatory variables of the birds were collected. The means obtained from the parameters evaluated were subjected to ANOVA with $5 \%$ of probability using SAS program. The temperature and moisture content (ITU) performed in the range of 71.65 to 68.49 during production time. There was no difference ( $p>0.05)$ for cloacal, skin and broilers mean temperatures at 7, 35 and 42 days of age fed with diets including corn DDGS. The use of corn DDG in broiler diets did not influence the thermoregulation of the broilers.

Keywords: Poultry. Coproducts. Body temperatures.

\section{Introdução}

O fator fibra é muito importante quando se trata de animais não ruminantes, tendo em vista que estes não possuem a capacidade de digestão de fibras. Portanto quanto maior o teor de fibra presente nos alimentos, maior a dificuldade de utilização nas dietas para animais. Isso ocorre porque a fibra em alta concentração diminui a energia metabolizável das rações, aumenta a taxa de passagem do alimento e diminui o aproveitamento dos nutrientes.

Segundo Ribeiro et al.(2008), o frango moderno tem pouca capacidade de termorregulação, sendo bem mais sensível ao calor do que ao frio. Desta forma, o incremento calórico, que é o calor gerado no processo de digestão, absorção e metabolismo dos nutrientes, pode afetar sua produção. Musharaf e Latshaw (1999) relatam que a proteína e a fibra proporcionam maior incremento calórico durante o metabolismo, em comparação à gordura e ao amido, o que pode aumentar a temperatura interna do animal e prejudicar seu desempenho.

A inclusão de grãos secos de destilaria (DDG - dried distillers grains) vem sendo reconhecidamente uma fonte de energia, proteína, vitaminas solúveis e minerais. É necessário determinar os níveis e alternativas de inclusão na alimentação de não ruminantes, visto que a composição de nutrientes e valor alimentício podem variar amplamente entre diferentes lotes de milho dentro de uma mesma usina de etanol e, também, entre diferentes usinas (Cremonez et al., 2015). O DDG apresenta grande concentração de lipídios, minerais, proteínas e, principalmente, carboidratos fibrosos.

O DDG de milho, comparado ao milho grão e ao farelo de soja, contém uma maior concentração de fibras, o amido do milho é utilizado na produção do etanol (Stein e Shurson, 2009) e o DDG, portanto, fica mais concentrado em relação a carboidratos fibrosos, caracterizando-se como coproduto de alta concentração de hemicelulose (Silva et al., 2016).

Este fator poderia ser um entrave em sua utilização, podendo causar prejuízos no desempenho animal. Alguns trabalhos mostram que a composição do ingrediente alimentar interfere diretamente no calor metabólico produzido no processo digestivo dos animais. Em vista do exposto, o objetivo do presente estudo foi determinar o efeito da inclusão de DDG de milho sobre os parâmetros de termorregulação em frangos de corte.

\section{Material e métodos}

O trabalho foi realizado no galpão para frangos de corte da Fazenda Experimental de Zootecnia e Agronomia da Universidade Federal de Mato Grosso, Campus Cuiabá. O projeto foi aprovado pelo comitê de ética no uso de animais da UFMT sob número 23108.227104/2017-13. O galpão, orientado no sentido leste-oeste, era equipado com sistema de cortinas externas nas laterais, acionamento mecânico tipo catracas, ventiladores 
e sistema de nebulização para controle de temperatura e umidade interna. O sistema de aquecimento foi realizado por meio de lâmpadas de infravermelho de $250 \mathrm{~W}$ de potência, instaladas individualmente em cada boxe experimental. $\mathrm{O}$ sistema de iluminação apresentava acendimento automático por meio de um timer, sem forros e cortina interna, e o telhado foi constituído de telhas de barro.

O galpão foi dividido em quatro linhas laterais, com 15 boxes telados de 2,5 $\mathrm{m}^{2}$ em cada linha; destes, foram utilizados 35 boxes, com densidade de 8,00 aves $/ \mathrm{m}^{2}$. O substrato utilizado para a cama foi de casca de arroz nova. O fornecimento de água e ração do $1^{\circ}$ ao $7^{\circ}$ dia foi realizado em copo de pressão e comedouro infantil, respectivamente. Para o período de 8 a 42 dias, o fornecimento de água ocorreu via bebedouro pendular e a ração em comedouros tubulares, com arraçoamento realizado duas vezes ao dia e reviragem diária da cama.

As temperaturas máximas e mínimas do galpão foram aferidas duas vezes ao dia, às $8 \mathrm{~h}$ e às $16 \mathrm{~h}$, e anotadas com o uso de termômetro de máxima e mínima. A média de temperaturas máxima e mínima, durante todo o período experimental, foi calculada pela soma da temperatura máxima ou mínima dividida pelo número de dias de alojamento.

O programa de iluminação foi executado de acordo com as recomendações do manual da linhagem Cobb (2012a). Utilizou-se delineamento inteiramente casualizado com cinco tratamentos, sendo quatro níveis e o tratamento controle, e sete repetições, totalizando 35 unidades experimentais com 20 aves em cada, totalizando 700 aves. As dietas experimentais foram à base de milho e farelo de soja e formuladas para atender às recomendações nutricionais de acordo com o manual da linhagem Cobb 500 (2012b).

A análise bromatológica do DDG de milho utilizado para formulação das rações está apresentada na Tabela 1.

Os tratamentos experimentais aplicados a partir do primeiro até o $42^{\circ}$ dia de idade (conforme a Tabela 2) foram: T1 - dieta controle com $0 \%$ de DDG; T2 - dieta com 4\% de DDG; T3 - dieta com $8 \%$ de DDG; T4 - dieta com 12\% de DDG; T5
- dieta com 16\% de DDG. Visando não prejudicar o desempenho dos frangos de corte, devido a fatores limitantes do uso de DDG como a quantidade de fibra e o teor de aminoácidos, estes valores foram baseados em uma revisão bibliográfica realizada sobre o tema em questão.

Tabela 1 - Análise bromatológica de grãos secos de destilaria (DDG) de milho

\begin{tabular}{lc}
\hline Análises & $\begin{array}{c}\text { Composição centesimal } \\
\text { na matéria mineral (\%) }\end{array}$ \\
\hline Proteína bruta (PB) & 42,73 \\
Extrato etéreo (EE) & 1,66 \\
Fibra bruta (FB) & 18,37 \\
Fibra detergente neutro (FDN) & 72,95 \\
Fibra detergente ácido (FDA) & 16,80 \\
Matéria mineral (MM) & 1,87 \\
Matéria seca (MS) & 89,00 \\
Extrato não nitrogenado (ENN) & 47,73 \\
NDT (estimado) & 79,09 \\
Cálcio (Ca) & 0,13 \\
Fósforo (P) & 0,53 \\
\hline
\end{tabular}

No final de cada fase de produção $(7,35$ e 42 dias de idade) foram coletados os dados relacionados às variáveis termorregulatórias das aves. A avaliação da temperatura corporal foi realizada às $13 \mathrm{~h}$, horário em que a temperatura interna e externa das aves é mais alta, utilizandose uma amostra de $10 \%$ das aves de cada parcela.

Foram coletados os dados das variáveis de temperatura da cabeça, canela, peito, dorso e asa utilizando-se termômetro infravermelho (Termômetro Digital Infravermelho Mira Laser), com mira laser a $15 \mathrm{~cm}$ de distância da pele do animal. Para a temperatura cloacal (TC), utilizouse termômetro clínico digital com ponta rígida (iColor-THGTH150B - Branco - G-Tech), introduzido na cloaca das aves, com emissão de sinal sonoro quanto à estabilização da temperatura. Para a avaliação da temperatura da cama, o termômetro infravermelho foi utilizado a $15 \mathrm{~cm}$ de distância do piso, até sua estabilização, em três pontos equidistantes da parcela experimental. 
Tabela 2 - Composição percentual das rações experimentais de frangos de corte de 1 a 7,8 a 35 e 36 a 42 dias de idade nos diferentes tratamentos

\begin{tabular}{|c|c|c|c|c|c|c|c|c|c|c|c|c|c|c|c|}
\hline \multirow{2}{*}{ Ingredientes (\%) } & \multicolumn{5}{|c|}{1 a 7 dias de idade } & \multicolumn{5}{|c|}{8 a 35 dias de idade } & \multicolumn{5}{|c|}{36 a 42 dias de idade } \\
\hline & T1 & T2 & T3 & T4 & T5 & T1 & T2 & T3 & T4 & T5 & T1 & T2 & T3 & T4 & T5 \\
\hline Milho & 51,20 & 49,70 & 48,20 & 46,80 & 45,40 & 54,91 & 53,88 & 52,90 & 51,46 & 50,36 & 59,17 & 58,1 & 57,0 & 55,58 & 54,44 \\
\hline Farelo de soja & 38,52 & 36,02 & 33,52 & 30,92 & 28,32 & 35,00 & 32,00 & 29,00 & 26,50 & 23,50 & 31,00 & 28,00 & 25,00 & 22,34 & 19,34 \\
\hline Calcário & 0,77 & 0,77 & 0,77 & 0,77 & 0,77 & 0,77 & 0,77 & 0,77 & 0,77 & 0,77 & 0,82 & 0,82 & 0,82 & 0,82 & 0,82 \\
\hline Fosfato bicálcico & 1,90 & 1,90 & 1,90 & 1,90 & 1,90 & 1,90 & 1,90 & 1,90 & 1,90 & 1,90 & 1,90 & 1,90 & 1,90 & 1,90 & 1,90 \\
\hline Sal comum & 0,55 & 0,55 & 0,55 & 0,55 & 0,55 & 0,55 & 0,55 & 0,55 & 0,55 & 0,55 & 0,55 & 0,55 & 0,55 & 0,55 & 0,55 \\
\hline Núcleo* & 1,80 & 1,80 & 1,80 & 1,80 & 1,80 & 1,80 & 1,80 & 1,80 & 1,80 & 1,80 & 1,80 & 1,8 & 1,8 & 1,80 & 1,80 \\
\hline Amido & 1,00 & 1,00 & 1,00 & 1,00 & 1,00 & 0,50 & 0,46 & 0,33 & 0,18 & 0,18 & 0,18 & 0,18 & 0,18 & 0,18 & 0,18 \\
\hline Óleo de soja & 3,51 & 3,51 & 3,51 & 3,51 & 3,51 & 3,90 & 3,90 & 3,90 & 3,90 & 3,90 & 3,90 & 3,90 & 3,90 & 3,90 & 3,90 \\
\hline DDG & 0,00 & 4,00 & 8,00 & 12,00 & 16,00 & 0,00 & 4,00 & 8,00 & 12 & 16,00 & 0,00 & 4,00 & 8,00 & 12,00 & 16,00 \\
\hline L-Treonina (98\%) & 0,03 & 0,03 & 0,03 & 0,03 & 0,03 & 0,03 & 0,05 & 0,10 & 0,15 & 0,20 & 0,01 & 0,06 & 0,11 & 0,16 & 0,21 \\
\hline DL - Metionina (99\%) & 0,57 & 0,57 & 0,57 & 0,57 & 0,57 & 0,57 & 0,57 & 0,57 & 0,57 & 0,57 & 0,57 & 0,57 & 0,57 & 0,57 & 0,57 \\
\hline L - Lisina (HCL) (78,84\%) & 0,15 & 0,15 & 0,15 & 0,15 & 0,15 & 0,07 & 0,12 & 0,18 & 0,22 & 0,27 & 0,10 & 0,12 & 0,17 & 0,20 & 0,26 \\
\hline \multirow{2}{*}{ Composição nutricional calculada } & \multicolumn{5}{|c|}{1 a 7 dias de idade } & \multicolumn{5}{|c|}{8 a 35 dias de idade } & \multicolumn{5}{|c|}{36 a 42 dias de idade } \\
\hline & T1 & T2 & T3 & T4 & T5 & T1 & T2 & T3 & T4 & T5 & T1 & T2 & T3 & T4 & T5 \\
\hline Energia metabolizável $(\mathrm{kcal} / \mathrm{kg})^{\star \star}$ & 3035 & 3035 & 3035 & 3035 & 3035 & 3108 & 3108 & 3108 & 3108 & 3108 & 3180 & 3180 & 3180 & 3180 & 3180 \\
\hline Proteína bruta (\%) & 22,0 & 22,0 & 22,0 & 22,0 & 22,0 & 20,00 & 20,00 & 20,00 & 20,00 & 20,00 & 19,00 & 19,00 & 19,00 & 19,00 & 19,00 \\
\hline Lisina digestível (\%) & 1,18 & 1,18 & 1,18 & 1,18 & 1,18 & 1,05 & 1,05 & 1,05 & 1,05 & 1,05 & 0,95 & 0,95 & 0,95 & 0,95 & 0,95 \\
\hline Metionina+Cistina digestível (\%) & 0,88 & 0,88 & 0,88 & 0,88 & 0,88 & 0,80 & 0,80 & 0,80 & 0,80 & 0,80 & 0,74 & 0,74 & 0,74 & 0,74 & 0,74 \\
\hline Triptofano digestível (\%) & 0,18 & 0,18 & 0,18 & 0,18 & 0,18 & 0,17 & 0,17 & 0,17 & 0,17 & 0,17 & 0,17 & 0,17 & 0,17 & 0,17 & 0,17 \\
\hline Treonina digestível (\%) & 0,77 & 0,77 & 0,77 & 0,77 & 0,77 & 0,69 & 0,69 & 0,69 & 0,69 & 0,69 & 0,65 & 0,65 & 0,65 & 0,65 & 0,65 \\
\hline Cálcio (\%) & 0,90 & 0,90 & 0,90 & 0,90 & 0,90 & 0,84 & 0,84 & 0,84 & 0,84 & 0,84 & 0,76 & 0,76 & 0,76 & 0,76 & 0,76 \\
\hline Fósforo disponível (\%) & 0,45 & 0,45 & 0,45 & 0,45 & 0,45 & 0,42 & 0,42 & 0,42 & 0,42 & 0,42 & 0,38 & 0,38 & 0,38 & 0,38 & 0,38 \\
\hline Sódio (\%) & 0,23 & 0,23 & 0,23 & 0,23 & 0,23 & 0,23 & 0,23 & 0,23 & 0,23 & 0,23 & 0,23 & 0,23 & 0,23 & 0,23 & 0,23 \\
\hline Fibra bruta (\%) & 2,96 & 3,09 & 3,21 & 3,33 & 3,46 & 2,84 & 3,13 & 3,41 & 3,70 & 3,99 & 2,69 & 2,99 & 3,27 & 3,56 & 3,85 \\
\hline
\end{tabular}

Nota: T1 - dieta controle com 0\% de grãos secos de destilaria (DDG); T2 - dieta com 4\% de DDG; T3 - dieta com 8\% de DDG; T4 - dieta com 12\% de DDG; T5 - dieta com 16\% de DDG * Fornece por kg de produto: Cálcio (máx) $210 \mathrm{~g} \mathrm{e} \mathrm{(min)} 170$ g, Fósforo (min) 50 g, Metionina (min) 22 g, vitamina A (min) 120000 UI, Vitamina D3 (min) 30000 UI, Vitamina E (min) 400 UI, Tiamina (B1) (min) 35 mg, Riboflavina (B2) (min) 130 mg, Piridoxina (B6) (min) 60 mg, Vitamina B12 (min) 300 mg, Vitamina K3 (min) 1600 mg, Zinco (min) 1380 mg, Cobre (min) 200 mg, Colina (min) 400 mg, Sódio (min) 26 g, Manganês (min) 160 mg, Ferro (min) 630 mg, lodo (min) 20 mg, Selênio (min) 6 mg, Fitase (min) 10000 FTU, Avilamicina 200 mg, Narasin + Navelbine $1000 \mathrm{mg}+1000 \mathrm{mg}$. ** Valores EMAn baseados na Tabela Europeia de Valores Energéticos de Alimentos para Aves (WPSA, 1989). 
Em seguida, os dados fisiológicos coletados foram utilizados para o cálculo da temperatura média da pele (TMP) e da temperatura média corporal (TMC) das aves, de acordo com a equação proposta por Richards (1971), considerando as temperaturas $\left({ }^{\circ} \mathrm{C}\right)$ de superfície e a temperatura retal das aves: TMP $=0,70 \mathrm{TD}+0,12 \mathrm{TA}+0,09 \mathrm{TCA}$ $+0,09 \mathrm{TP}$, em que TD = temperatura do dorso, $\mathrm{TA}=$ temperatura da asa, TCA = temperatura da cabeça, $\mathrm{TP}=$ temperatura da canela; $\mathrm{TMC}=0,3 \mathrm{TMP}+0,7$ $\mathrm{TC}$, em que TC = temperatura clocal.

O índice de temperatura e umidade (ITU) foi calculado a partir do modelo indicado por Thom (1959), conforme descrito na equação abaixo, sendo $\mathrm{T}=$ temperatura do $\operatorname{ar}\left({ }^{\circ} \mathrm{C}\right)$ e UR $=$ umidade relativa do $\operatorname{ar}(\%)$ :

$$
\mathrm{ITU}=\left(08 x \mathrm{TA}+\left(\frac{\mathrm{UR}}{100}\right) x \mathrm{TA}-14,4+46,4\right)
$$

As variáveis estudadas foram submetidas às premissas estatísticas de normalidade de resíduos através doteste de Shapiro Wilke a homogeneidade das variâncias por meio do teste de Levene's. Os dados que apresentaram disparidade passaram por transformação e, em seguida, realizou-se a análise de variância através do programa $R$ Studio Ink ${ }^{\circledR}$. As médias foram submetidas ao teste de Tukey ao nível de $5 \%$ de probabilidade, utilizandose o programa SAS (1990).

\section{Resultados e discussão}

As temperaturas máxima e mínima e a umidade relativa do ar, verificadas diariamente durante o experimento às $8 \mathrm{~h}$ e às $16 \mathrm{~h}$, estão apresentadas na Tabela 3.

As condições de conforto térmico até as primeiras duas semanas de vida dos frangos de corte são de $33^{\circ} \mathrm{C}$ reduzindo até $24^{\circ} \mathrm{C}$, com quatro semanas de idade, e para 21 a $22{ }^{\circ} \mathrm{C}$ com seis semanas de idade, e a umidade relativa do ar em torno de $57 \%$ a $69 \%$ (Oliveira, 2016). Conforme os valores registrados pelos termômetros, observase que as aves passaram por estresse térmico por calor no período total de produção.
Com a finalidade de determinar os níveis de conforto frente às condições ambientais às quais os animais estão sujeitos, diversos índices como a temperatura efetiva, índice de temperatura e umidade (ITU) e índice de temperatura de globo e umidade (ITGU) foram desenvolvidos (Hahn, 1985).

O ITU esteve na faixa de 68,49 a 71,65 durante o tempo de produção. Segundo Araujo et al. (2017), o ITU é um valor considerado adimensional, em que valores de até 74 representam ambientes confortáveis, de 74 a 78 exigem cuidado no manejo, de 79 a 84 são perigosos e de 85 em diante apresentam condições extremas de estresse, podendo elevar a mortalidade do plantel.

Não houve efeito $(p>0,05)$ para as variáveis temperatura cloacal, temperatura média corporal, temperatura média da pele, e temeratura média da cama de frangos de corte aos 7, 35 e 42 dias de idade, alimentados com dietas com diferentes níveis de DDG de milho (Tabela 4).

Segundo Brown-Brandt et al. (2003), a temperatura cloacal representa a temperatura do núcleo corporal e pode ser utilizada para avaliar a condição de conforto ou estresse. Han et al. (2010) relatam que frangos de corte, quando expostos à temperatura ambiental acima de $25^{\circ} \mathrm{C}$, apresentam temperaturas da cloaca e da pele significativamente aumentadas.

Conforme relatado por Marchini et al., (2007) a temperatura retal de frangos em ambientes termoneutros é de $41,4{ }^{\circ} \mathrm{C}$. Na primeira semana, os valores de temperatura cloacal permaneceram acima do preconizado pela literatura, estando entre 42,14 e $42,48 C^{\circ}$. Já a temperatura média corporal variou de 40,72 a $41,22^{\circ} \mathrm{C}$ e a temperatura média da pele de 37,42 a $38,35^{\circ} \mathrm{C}$.

O pintinho possui grande relação entre área/ volume corporal, dificultando o processo de retenção de calor pela ave. $O$ desconforto térmico pode acarretar sérios problemas na produção como menor taxa de crescimento, maior consumo de água, aceleração do ritmo cardíaco e alteração da conversão alimentar. Cordeiro et al. (2010) também relatam que quando a temperatura está acima do conforto térmico das aves pode ocorrer desidratação, o que prejudica o consumo de ração e o ganho de peso. 
Tabela 3 - Valores da temperatura máxima e mínima e umidade relativa do ar registrados no interior da instalação nas fases de produção 7, 35 e 42 dias de idade

\begin{tabular}{|c|c|c|c|c|c|c|}
\hline & Manhã & Tarde & Manhã & Tarde & Manhã & Tarde \\
\hline & \multicolumn{2}{|c|}{7 dias de idade } & \multicolumn{2}{|c|}{35 dias de idade } & \multicolumn{2}{|c|}{42 dias de idade } \\
\hline Temperatura máxima $\left({ }^{\circ} \mathrm{C}\right)$ & 31,60 & 36,52 & 29,70 & 35,65 & 29,64 & 34,55 \\
\hline Umidade máxima (\%) & 82,50 & 80,20 & 83,50 & 81,62 & 79,45 & 78,82 \\
\hline Temperatura mínima $\left({ }^{\circ} \mathrm{C}\right)$ & 21,31 & 25,64 & 22,33 & 26,67 & 20,40 & 21,32 \\
\hline Umidade mínima (\%) & 44,32 & 21,23 & 43,32 & 26,56 & 45,56 & 27,61 \\
\hline Temperatura média $\left({ }^{\circ} \mathrm{C}\right)$ & \multicolumn{2}{|c|}{28,76} & \multicolumn{2}{|c|}{28,58} & \multicolumn{2}{|c|}{26,47} \\
\hline Umidade média(\%) & \multicolumn{2}{|c|}{57,06} & \multicolumn{2}{|c|}{58,75} & \multicolumn{2}{|c|}{57,86} \\
\hline Índice de temperatura e umidade & \multicolumn{2}{|c|}{71,41} & \multicolumn{2}{|c|}{71,65} & \multicolumn{2}{|c|}{68,49} \\
\hline
\end{tabular}

Tabela 4 - Temperatura cloacal (TC), temperatura média corporal (TMC), temperatura média da pele (TMP) e temperatura média da cama (TCA) de frangos de corte aos 7, 35 e 42 dias de idade, alimentados com dietas com níveis de grãos secos de destilaria (DDG) de milho

\begin{tabular}{|c|c|c|c|c|c|c|c|}
\hline \multirow[b]{2}{*}{ Parâmetros } & \multicolumn{5}{|c|}{ Níveis de DDG de milho (\%) } & \multirow[b]{2}{*}{ CV (\%) } & \multirow[b]{2}{*}{ p-valor } \\
\hline & 0 & 4 & 8 & 12 & 16 & & \\
\hline \multicolumn{8}{|c|}{7 dias de idade } \\
\hline $\mathrm{TC}\left({ }^{\circ} \mathrm{C}\right)^{\mathrm{ns}}$ & 42,34 & 42,48 & 42,18 & 42,32 & 42,14 & 1,29 & 0,731 \\
\hline $\operatorname{TMC}\left({ }^{\circ} \mathrm{C}\right)^{\text {ns }}$ & 41,14 & 41,22 & 40,93 & 40,97 & 40,72 & 1,81 & 0,288 \\
\hline $\operatorname{TMP}\left({ }^{\circ} \mathrm{C}\right)^{\text {ns }}$ & 38,35 & 38,30 & 38,02 & 37,82 & 37,42 & 1,74 & 0,083 \\
\hline $\operatorname{TCA}\left({ }^{\circ} \mathrm{C}\right)^{\text {ns }}$ & 30,55 & 30,28 & 31,03 & 30,76 & 31,45 & 1,89 & 0,398 \\
\hline \multicolumn{8}{|c|}{35 dias de idade } \\
\hline $\mathrm{TC}\left({ }^{\circ} \mathrm{C}\right)^{\mathrm{ns}}$ & 39,42 & 39,01 & 39,67 & 39,41 & 39,68 & 4,98 & 0,663 \\
\hline $\operatorname{TMC}\left({ }^{\circ} \mathrm{C}\right)^{\text {ns }}$ & 38,99 & 38,68 & 39,15 & 39,08 & 39,26 & 1,24 & 0,288 \\
\hline $\operatorname{TMP}\left({ }^{\circ} \mathrm{C}\right)^{\text {ns }}$ & 38,00 & 37,92 & 37,95 & 38,30 & 38,28 & 4,89 & 0,991 \\
\hline $\operatorname{TCA}\left({ }^{\circ} \mathrm{C}\right)^{\text {ns }}$ & 32,14 & 31,99 & 32,65 & 32,77 & 32,12 & 1,24 & 0,563 \\
\hline \multicolumn{8}{|c|}{42 dias de idade } \\
\hline $\mathrm{TC}\left({ }^{\circ} \mathrm{C}\right)^{\mathrm{ns}}$ & 40,64 & 39,45 & 39,47 & 39,32 & 40,44 & 5,87 & 0,766 \\
\hline $\operatorname{TMC}\left({ }^{\circ} \mathrm{C}\right)^{\text {ns }}$ & 39,60 & 38,81 & 38,95 & 38,68 & 39,64 & 2,12 & 0,367 \\
\hline $\operatorname{TMP}\left({ }^{\circ} \mathrm{C}\right)^{\mathrm{ns}}$ & 37,20 & 37,34 & 37,76 & 37,21 & 37,78 & 2,69 & 0,764 \\
\hline $\operatorname{TCA}\left({ }^{\circ} \mathrm{C}\right)^{\mathrm{ns}}$ & 31,44 & 31,92 & 31,92 & 31,48 & 32,15 & 1,48 & 0,434 \\
\hline
\end{tabular}

Nota: CV = coeficiente de variação; ${ }^{\text {ns }}=$ não significativo.

Aos 35 dias, as médias de TC, TMC e TMP variaram em 39,01 e $39,42{ }^{\circ} \mathrm{C}, 38,68$ e $39,265^{\circ} \mathrm{C}$ e 37,92 e $38,30{ }^{\circ} \mathrm{C}$, respectivamente. Alves (2012) relata que várias medidas podem ser tomadas para minimizar o desconforto térmico. A utilização de certos alimentos na dieta que forneçam energia com baixo incremento calórico pode ser uma destas medidas. Animais em períodos de estresse térmico reduzem a alimentação em função da necessidade de diminuir seu incremento calórico.

Segundo Musharaf e Latshaw (1999), em comparação aos lipídios, a fibra e a proteína possuem maior incremento calórico. Blaxter (1989) afirma que o incremento calórico é o aumento 
na produção de calor associado ao consumo de alimento. Este termo é advindo da diferença de calor produzido por um animal comparando antes e após alimentar-se, e isto é totalmente dependente da quantidade e do tipo de alimento consumido.

O DDG de milho, comparado ao milho grão e ao farelo de soja, contém uma maior concentração de fibras, onde se atingem valores de FDA superiores a $10 \%$ e de FDN acima de $30 \%$ (Stein e Shurson, 2009). Este fator poderia ser um entrave em sua utilização, podendo causar prejuízos no desempenho animal. Alguns trabalhos mostram que a composição do ingrediente alimentar interfere diretamente no calor metabólico produzido no processo digestivo dos animais.

Ferreira et al. (2011), avaliando a variação de produção de calor de pintinhos alimentados com diferentes densidades energéticas, observaram que o aumento da densidade energética da dieta proporcionou menor temperatura média superficial. Sarmiento-Franco et al. (2000) relataram que alimentos fibrosos proporcionaram maior incremento calórico em frangos, e propuseram que as diferenças no efeito térmico são devido a efeitos fisiológicos e mecânicos resultantes da variação da fonte de fibra sobre o trato digestório das aves. Miranda et al. (2017) verificaram que utilização do farelo de algodão em substituição à proteína do farelo de soja não altera a temperatura da pele e temperatura superficial de frangos de corte, o que corrobora com a presente pesquisa.

\section{Conclusão}

Os parâmetros termorregulatórios dos frangos de corte não sofreram interferência dos níveis de inclusão de grãos secos de destilaria na dieta.

\section{Referências}

Alves FMS. Calor metabólico de frangos de corte e poedeiras alimentados com diferentes fontes lipídicas [dissertação]. Dourados, MS: Universidade Federal da Grande Dourados; 2012. 45 p.
Araujo JA, Monção AF, Vieira RKR. Avaliação bioclimática para frangos de corte na época das chuvas na região sudeste do Estado do Pará. Rev Agroecossist. 2017;9(1):180-8.

Blaxter K. Energy metabolism in animals and man. Cambridge: Cambridge University Press; 1989. 352 p.

Brown-Brandt TM, Yanagi Jr T, Xin H, Gates RS, Bucklin RA. A new telemetry system for measuring core body temperature in livestock and poultry. Appl Eng Agric. 2003;19(5):583-9.

Cobb. Manual de Manejo de Frangos de Corte. São Paulo: Cobb Vantress Brasil; 2012a. 47 p.

Cobb 500 Suplemento de Crescimento e Nutrição para Frangos de Corte. São Paulo: Cobb Vantress Brasil; $2012 b$.

Cordeiro MB, Tinôco IFF, Silva JN, Vigoderis RB, Pinto FAC, Cecon PR. Conforto térmico e desempenho de pintos de corte submetidos a diferentes sistemas de aquecimento no período de inverno. R Bras Zootec. 2010;39(1):217-24.

Cremonez PA, Feroldi M, Nadaleti WC, Rossi E, Feiden A, Camargo MP, et al. Biodiesel production in Brazil: current scenario and perspectives. Renew Sust Energ Rev. 2015;42:415-28.

Ferreira VMOS, Francisco NS, Belloni M, Aguirre GMZ, Caldara FR, Nääs IA, et al. Infrared thermography applied to the evaluation of metabolic heat loss of chicks fed with different energy densities. Rev Bras Cienc Avic. $2011 ; 13(2): 113-8$.

Hahn GL. Management and housing of farm animals in hot environments. In: Yousef MK. Stress physiology in livestock. v 2. Boca Raton, FL: CRC Press; 1985. p.15174.

Han AY, Zhang MH, Zuo XL, Zheng SS, Zhao CF, Feng JH, et al. Effect of acute heat stress on calcium concentration, proliferation, cell cycle, and interleukin-2 production in splenic lymphocytes from broiler chickens. Poult Sci. 2010;89(10):2063-70. 
Marchini CFP, Silva PL, Nascimento MRBM, Tavares M. Frequência respiratória e temperatura cloacal em frangos de corte submetidos à temperatura ambiente cíclica elevada. Arch Vet Sci. 2007;12(1):41-6.

Miranda LMB, Goulart CC, Leite SCB, Batista ASM, Lima RC. Farelo de algodão em dietas com ou sem suplementação de enzimas para frango de corte. Rev Cienc Agron. 2017;48(4):690-9.

Musharaf NA, Latshaw JD. Heat increment as affected by protein and amino acid nutrition. Worlds Poult Sci J. 1999;55(3):233-40.

Oliveira AP. Caracterização do consumidor de carne de frango em Júlio Borges-PI. Rev Cient Prod Anim. 2016;17(2):129-41.

Ribeiro AML, Vogt LK, Canal CW, Laganá C, Streck AF. Suplementação de vitaminas e minerais orgânicos e sua ação sobre a imunocompetência de frangos de corte submetidos a estresse por calor. Rev Bras Zootec. 2008;37(4):636-44.
Richards SA. The significance of changes in the temperature of the skin and body core of the chicken in the regulation of heat loss. J Physiol. 1971;216(1):1-10.

SAS Institute. SAS/STAT User's guide: Version 6, Vol. 2. Cary, NC: SAS Institute Incorporated; 1990.

Sarmiento-Franco L, MacLeod MG, McNab JM. True metabolisable energy, heat increment and net energy values of two high fibre foodstuffs in cockerels. Br Poult Sci. 2000;41(5):625-9.

Silva JR, Netto DP, Scussel VM. Grãos secos de destilaria com solúveis, aplicação em alimentos e segurança - uma revisão. Pubvet. 2016;10(3):190-270.

Stein $\mathrm{HH}$, Shurson GC. Board-invited review: The use and application of distillers dried grains with solubles in swine diets. J Anim Sci. 2009;87(4):1292-303.

World's Poultry Science Association. Nutrition of the European Federation of Branches. Beekbergen, Netherlands: WPSA; 1989. 